\title{
CARCINOMA DE LA VULVA
}

\section{Análisis de 17 casos.}

\author{
Dr. Alfonso Jubiz H.*
}

HOSPITAL UNIVERSITARIO SAN VICENTE DE PAUL - Medellín, 1975.

\section{Introducción}

Los tumores malignos de la vulva no se ven favorecidos paar su estudio porque su baja frecuencia le ha restado importancia en relación a los otros tumores del aparato genital femenino. Se acepta que el $3.4 \%$ de todos los cánceres del aparato genital femenino corresponden a la vulva y es el tercero de los de la pelvis femenina (7).

Si tenemos en cuenta que la mayoría de los casos se presentan en pacientes de edad avanzada, el diagnóstico temprano no es lo común ya que estas pacientes por un falso pudor le restan importancia a las lesiones iniciales de la enfermedad. Sin embargo, en una tercera parte de los casos es el médico el culpable de esta situación por no dar a las lesiones ulcerativas y vegetantes de la vulva el valor real que se merecen y demorar el estudio histopatológico de las mismas (8). Fue Taussig en 1940 quien primero hizo énfasis en la cirugía radical del carcinoma de la vulva y analiza 155 casos (9). En Colombia, López Escobar en 1951 describe con lujo de detalles la técnica de Basset y presenta la estadística de los casos estudiados en el Instituto Nacional del Radium desde su fundación hasta Septiembre de 1950 (6). Los artícu- los de la literatura médica colombiana analizan diferentes aspectos y han enriquecido el arsenal bibliográfico de nuestra ginecología $(4,2,5,1)$.

Nuestra casuística no es muy abundante pero mi predilección por el tema y el convencimiento de las ventajas de la cirugía en estos tumores me movieron a revisar los casos ocurridos en el Hospital Universitario San Vicente de Paúl.

\section{Material y Métodos}

En el Departamento de Historias Médicas y Archivo del Hospital Universitario San Vicente de Paúl se encontraron 17 historias con el diagnóstico de carcinoma de la vulva incluyendo el carcinoma intraepitelial. Estas historias corresponden a los años 1970 a 1974,14 al servicio general y 3 al servicio de pensionado.

Se analizaron las siguientes variables: edad de la paciente, procedencia, paridad, evolución de la enfermedad en meses, síntoma inicial, diagnóstico histopatológico, clasificación clínica, tratamiento y el estado final de la paciente hasta el 30 de Septiembre de 1975.

* Profesor I. Departamento de Obstetricia y Ginecología. Facultad de Medicina Universidad de Antioquia. 
La clasiifcación del carcinoma de la vulva en T.N.M. adoptada por la FIGO desde Enero 1: de 1971 no pudo ser utilizada porque los casos estudiados se clasiifcaron en grados ya que durante el tiempo en que se presentaron no había mucha aceptación de la primera clasificación. El escaso número de casos no nos permite hacer un estudio estadístico conclusivo, pero sí un análisis de las variables antes anotadas.

\section{Resultados}

Motivo de consulta: La presencia de masa de 2 a más de $20 \mathrm{cms}$. fue el síntoma más frecuente encontrado en 13 pacientes, el prurito estuvo acompañando a los tres carcinomas intraepiteliales. La masa se encontró en el lado izquierdo en 9 casos, en el lado derecho en 3 y en las restantes la masa era tan grande que ocupaba toda la zona vulvar y perineal.

Edad: La edad promedio de las pacientes fue de 55 años, con edades extremas de 30 y 84 años. Es aceptado que la lesión aparece más frecuentemente en las edades avanzadas y especialmente en la postmenopausia, donde la atrofia de los tejidos vulvares por la falla ovárica, la vulvitis y la leucoplasia son hallazgos constantes.

Procedencia: Todas las pacientes procedían del Departamento de Antioquia, 8 de la ciudad de Medellín y las restantes de otros municipios del Departamento. Es posible que este hecho haya demorado la primera consulta de la paciente, ya que 7 de éstas tenían estados avanzados.

Paridad: Solamente 3 pacientes son multigestantes. Green Ulfelder y Meigs, citado por Gartner (9) consideran que como ocurre en el carci- noma endometrial, el de la vulva es más frecuente en solteras y nuligestantes.

Evolución de la enfermedad: Es muy difícil saber exactamente cuándo se inició la lesión maligna. Se encontró una paciente con una evolución de 3 meses con un estadio III, 7 pacientes tenían una evolución mayor de 12 meses y se encontraron 2 con una historia de prurito vulvar de 60 meses.

\section{Edad, Diagnóstico histopatológico, Clasificación, Tratamiento y Estado actual: Cuadros Nos. 1-A y 1-B.}

En 13 casos el diagnóstico histopatológico mostró un carcinoma epidermoide espino-celular, hallazgo que confirma su frecuencia elevada en los tumores malignos de la vulva. En dos casos el diagnóstico fue de carcinoma epidermoide verrucoso, variedad ésta, que tiene asiento en lesiones condilomatosas. En una paciente se encontró melanoma con una lesión similar en el cuello uterino, en la otra paciente no fue posible conocer eldi agnóstico histopatológico.

En 9 casos el estadio clínico fue avanzado (III y IV). La vulvectomía radical con linfadenectomía fue el tratamiento de elección sin tener en cuenta la edad de la paciente. Es de anotar que la paciente sometida a extirpación del labio menor y mayor derecho era de servicio privado. En 2 pacientes no fue posible practicar ningún tratamiento quirúrgico.

A pesar de encontrar 6 pacientes en buenas condiciones al terminar la revisión de las historias no es posible hacer una evaluación real del tratamiento y seguimiento toda vez que sólo una paciente lleva 60 meses de operada y se encuentra bien. 


\section{CUADRO NN 1-A}

ANALISIS DE 17 CASOS

\begin{tabular}{|c|c|c|c|c|c|}
\hline $\begin{array}{l}\text { Edad en } \\
\text { años }\end{array}$ & $\begin{array}{l}\text { Diagnóstico } \\
\text { histopatológico }\end{array}$ & $\begin{array}{l}\text { Clasific } \\
\text { clínica }\end{array}$ & ación & Tratamiento & $\begin{array}{l}\text { Estado actual } \\
\text { de la paciente }\end{array}$ \\
\hline 30 & $\begin{array}{l}\text { Ca. Epidermoide } \\
\text { Intraepitelial }\end{array}$ & Estado & 0 & $\begin{array}{l}\text { Vulvectomía Radical. } \\
\text { Linfadenectomía }\end{array}$ & Bien \\
\hline 51 & $\begin{array}{l}\text { Ca. Epidermoide } \\
\text { Intraepitelial }\end{array}$ & & 0 & Vulvectomía Simple & Bien \\
\hline 53 & $\begin{array}{l}\text { Ca. Epidermoide } \\
\text { Intraepitelial }\end{array}$ & & 0 & Vulvectomía & Bien \\
\hline 84 & $\begin{array}{l}\text { Ca. Epidermoide } \\
\text { Espinocelular }\end{array}$ & & 1 & Vulvectomía Radical & $\begin{array}{l}\text { Murió a los } 15 \\
\text { días postoperatorio }\end{array}$ \\
\hline 45 & Ca. Epidermoide & & 1 & $\begin{array}{l}\text { Extirpación Labio mayor } \\
\text { y menor derecho }\end{array}$ & Desconocido \\
\hline 40 & $\begin{array}{l}\text { Ca. Epidermoide } \\
\text { Verrucoso }\end{array}$ & & 11 & $\begin{array}{l}\text { Vulvectomía Radical. } \\
\text { Colostomía }\end{array}$ & Bien \\
\hline 57 & $\begin{array}{l}\text { Ca. Epidermoide } \\
\text { Espinocelular }\end{array}$ & & 11 & $\begin{array}{l}\text { Vulvectomía Radical. } \\
\text { Linfadenectomía }\end{array}$ & Desconocido \\
\hline 35 & $\begin{array}{l}\text { Ca. Epidermoide } \\
\text { Espinocelular }\end{array}$ & & 11 & $\begin{array}{l}\text { Vulvectomía Radical. } \\
\text { Linfadenectomía }\end{array}$ & Bien \\
\hline
\end{tabular}

\section{CUADRO NN 1-B}

\begin{tabular}{|c|c|c|c|c|c|}
\hline $\begin{array}{l}\text { Edad en } \\
\text { años }\end{array}$ & $\begin{array}{l}\text { Diagnóstico } \\
\text { histopatológico }\end{array}$ & $\begin{array}{l}\text { Clasific } \\
\text { clínica }\end{array}$ & ción & Tratamiento & $\begin{array}{l}\text { Estado actual } \\
\text { de la paciente }\end{array}$ \\
\hline 77 & $\begin{array}{l}\text { Ca. Epidermoide } \\
\text { Espinocelular }\end{array}$ & Estado & III & Vulvectomía Simple & Muerta \\
\hline 63 & $\begin{array}{l}\text { Ca. Epidermoide } \\
\text { Espinocelular }\end{array}$ & & 111 & $\begin{array}{l}\text { Vulvectomía Radical. } \\
\text { Linfadenectomía }\end{array}$ & Muerta \\
\hline 57 & $\begin{array}{l}\text { Ca. Epidermoide } \\
\text { Espinocelular }\end{array}$ & & 111 & $\begin{array}{l}\text { Vulvectomía Radical. } \\
\text { Linfadenectomía }\end{array}$ & Desconocido \\
\hline 49 & $\begin{array}{l}\text { Ca. Epidermoide } \\
\text { Espinocelular }\end{array}$ & & III & $\begin{array}{l}\text { Vulvectomía Radical. } \\
\text { Linfadenectomía }\end{array}$ & Desconocido \\
\hline 60 & $\begin{array}{l}\text { Ca. Epidermoide } \\
\text { Verrucoso }\end{array}$ & & III & $\begin{array}{l}\text { Vulvectomía Radical. } \\
\text { Linfadenectomía }\end{array}$ & Desconocido \\
\hline 70 & $\begin{array}{l}\text { Ca. Epidermoide } \\
\text { Inmaduro }\end{array}$ & & IV & Sin Tratamiento & Desconocido \\
\hline 53 & Melanoma & & IV & Radium Quimioterapia & Muerta \\
\hline 48 & $\begin{array}{l}\text { Ca. Epidermoide } \\
\text { Espinocelular }\end{array}$ & & IV & $\begin{array}{l}\text { Vulvectomía Radical. } \\
\text { Linfadenectomía. } \\
\text { Exanteración anterior }\end{array}$ & Bien \\
\hline 60 & $\begin{array}{l}\text { Ca. Epidermoide } \\
\text { Espinocelular }\end{array}$ & & IV & Sin Tratamiento & Desconocido \\
\hline
\end{tabular}

\section{Comentarios}

Es indudable que la incidencia del carcinoma de la vulva aumenta con la edad de la paciente. El diagnóstico diferencial temprano y oportuno permite ofrecer a estas pacientes una cirugía radical como única esperanza de curación. Es muy importante estudiar cuidadosamente las lesiones vulvares y practicar biopsias más frecuentes en condilomas acuminados y leucoplasias, las cuales pueden disimular la enfermdead. 
Teniendo en cuenta el origen multicéntrico del carcinoma de la vulva y el sistema de linfáticos pequeños abundantes, no tienen cabida las cirugías parciales.

A pesar de encontrar $1 / 3$ de los ganglios palpables sin metástasis, se recomienda la linfadenectomía de regiones inguinales, femorales, incluyendo el ganglio de Cloquet, ilíacos, obturadores e hipogástricos, ya que en la tercera parte de los casos con metástasis no se palparon los ganglios. Cuando hay metástasis el futuro es sombrío. Sólo 2 de las pacientes tenían metástasis ganglionares.

La cirugía debe ser tan amplia que no permita el cierre como lo sostiene Way (3). Collins considera que la curabilidad va paralela a la amplitud de la resección. Es muy importante explicar muy bien a las pacientes el procedimiento a seguir y practicar evaluación psiquiátrica preoperatoria. Una de las pacientes presentó en el post-operatorio una úlcera gástrica aguda. El post-operatorio es largo, tormentoso y exigente para la paciente y para el médico.

\section{Resumen y Conclusiones}

Se analizan 17 casos de carcinoma de la vulva en el Hospital Universitario San Vicente de Paúl durante los años 1970 a 1974.

La edad promedio fue de 55 años. Dos terceras partes $(2 / 3)$ de las pacientes eran grandes multíparas. En la mitad de los casos la clasificación clínica correspondió a estadios avanzados, grados III y IV. La evolución de la lesión fue variable y en 2 se encontró como único síntoma el prurito vulvar de 5 años. El diagnóstico anatomopatológico correspondió al carcinoma epidermoide espinocelular en 13 casos, epidermoide verrucoso en 2, melanoma en uno y en el otro no se conoció el diagnóstico histológico.

En 10 pacientes se practicó vulvectomía radical con linfadenectomía, en una de ellas con exanteración anterior y en otra con colostomía. Vulvectomía simple se efectuó en 3 casos, no se practicó ningún tratamiento en 2, radium y quimioterapia en una, en la restante sólo se practicó resección de la lesión. Cuatro pacientes han muerto, seis se encuentran en buenas condiciones, en las restantes no fue posible obtener información de su estado, pero es de suponer que murieron por lo avanzado de su estadio clínico. El seguimiento mínimo fue de 4 meses y el máximo de 60 .

\section{Conclusiones}

1. No fulgurar lesiones vegetantes de vulva sin biopsia previa.

2. No utilizar RX para tratar lesiones pruriginosas de la vulva.

3. La cirugía radical con linfadenectomía es el tratamiento de elección cuando las condiciones de la paciente lo permiten.

4. En estadios avanzados sin metástasis extrapélvicas, la exanteración anterior, posterior o total es la única posibilidad de curación.

5. Debe utilizarse la clasificación clínica aceptada por la FIGO desde Enero 10 de 1971.

\section{Summary}

17 cases of Vulva's Carcinoma were analysed during the period of 1970 to 1974 at the Hospital Universitario San Vicente de Paúl.

The mean age was 55 years. Two thirds $(2 / 3)$ of these were Great Multiparas. In half of the cases the 
Clinic clasification corresponded to advanced stages, (III \& IV).

The evolution of the lesion was variable and in 2 cases the only sign was Vulvar Pruritus during 5 years. The Anatomopathologyc Diagnostic was Espinocelular Epidermoid Carcinoma in 13 cases, Verrucoss Epidermoid Carcinoma in 2 cases, Melanoma in one and in the other the Histological Diagnostic was unknown.

In 10 patients a Radical Vulvectomy with Linfadenectomy, was carried out; In one of them with Anterior Extension and another one with Colostomy. Simple Vulvectomy was carried out in 3 cases, no treatment in two, Radium and Chemotherapy in one, Simple Resection of the lesion in one.

4 patients die, six are in good conditions, and the rest it was it was impossible to obtain information, but we suppose they are dead, since they had very advanced disease. The minimal follow up was 4 months and the maximal 60.

\section{Conclussions}

1. Vegetant Vulvar lesion are not to be Fulgurated without previous Biopsy.

2. Radical Therapy is not to be used for Pruriginous Vulvar Lesions.

3. Radical Surgery with Linphadenecthomy is the best treatment when the patient's conditions permit it.
4. Advance stages without Extra Pelvis Metastatic disease Anterior Exantenation Posterial or Total is the only possible curative therapy.

5. The Clinical clasification accepted by the FIGO in 1971 must be used.

\section{BIBLIOGRAFIA}

1 AMAYA LEON, $H$. Carcinoma vulvar asociado a tuberculosis. Revista Fac. Med. Bogotá 17 : $490,1949$.

2 DUARTE CONTRERAS, A. y cols. Cáncer de la vulva. Rev. Col. Obst. y Ginec. 21: 233248, Mayo-Junio, 1970.

3 GARDNER, H. L. Tumores vulvares y vaginales. Clin. Obst. y Ginec. Pág. 1052, Diciembre, 1965.

4 GIANNUZZI, P. HERNANDEZ BARRETO, A. Carcinoma de vulva con tratamiento radical. Informe de 2 casos. Rev. Soc. Med. Quir. Barranquilla, 4: 38-52, Marzo 1960.

5 JORDAN, G. y cols. Cáncer de la vulva. Rev. Col. Obst. Ginec. 16: 435-438. NoviembreDiciembre, 1965.

6 LOPEZ ESCOBAR. G. ORJUELA, R. Tumores malignos de la vulva. Rev. Col. Obst. y Ginec. 21: 211, 1951 y 3: 9, 1952.

7 NOVAK, E., SEEGAR JONES, G. W. JONES, H. Tratado de Ginecología. Ed. Interamericana, México, Octava Edición, 1971.

8 PARSONS, L. and SOMMERS, S. Gynecology, W. B. Saunders Co. Philadelphia and London 1a. Ed. 1963.

9 TAUSSIG, F. J. Cancer of the vulva. Analysis of 156 casos. Obst. and Gynec. 40: 764, 1940. 